\section{Low Voltage Achieves Large Strain in Electroreactive Polymer}

Soft polymeric materials that can convert electrical energy into mechanical energy have become an intensive area of study recently, owing to their potential to mimic biological responses. Mohsen Shahinpoor of the Artificial Muscle Research Institute (AMRI) at the University of New Mexico and Kwang Kim from the Active Materials and Processing Laboratory (AMPL) of the University of Nevada have combined lithium-doped poly(ethylene oxide) (PEO) with the plasticizer poly(ethylene glycol) (PEG) to fabricate 320- $\mu$ m-thick actuators exhibiting large strain $(>1 \%$ bending strain) at a relatively low applied electric field $(<10 \mathrm{~V} / \mathrm{mm})$. Actuator motion in these materials arises from the cation attraction to the anode relative to the polymer host, which creates a pressure gradient leading to deformation. This deformation is reversible with the direction of the electric field and also displays stable operation after more than 10 million cycles in air.

As reported in the May 6 issue of Applied Physics Letters, the actuation of ionic conductive polymers such as PEO is increased by the use of the plasticizer PEG, which decreases the crystallinity of the medium, leading to increased ion mobility. Load and force measurements indicate that the polymer solid-state actuator produces a useful force density of up to $20 \mathrm{~g}$-force/g of PEO-PEG polymer in a cantilevered configuration, under an applied voltage of 1-3 V, while maintaining a constant frequency of $0.5 \mathrm{~Hz}$. Furthermore, the researchers report that strain power-density variations for the PEO-PEG solid-state actuators indicate that the current $2 \%$ strain values can lead to power densities of over $50 \mathrm{~J} / \mathrm{kg}$, while further improvement of these actuators to enable them to generate $4 \%$ strain would lead to power densities of 200 $\mathrm{J} / \mathrm{kg}$, comparable to biological muscles.

KYLE BRINKMAN

\section{D Imaging of Materials}

Achieved with Differential-Aperture X-Ray Microscopy

X-ray diffraction provides two-dimensional (2D) information on the structure, orientation, and distortion of a crystal. However, information along the path of the incident beam is hidden within the superposition of patterns and cannot be discerned. A group of researchers from Oak Ridge National Laboratory have obtained depth-resolved three-dimensional (3D) images of crystals using a technique they call differential-aperture $x$-ray microscopy (DAXM).

As reported in the February 21 issue of Nature, B.C. Larson and colleagues incorporated an x-ray-absorbing wire as a knife-edge depth profiler. The wire is moved stepwise across the diffraction patterns, selectively blocking out diffracted $x$-rays from reaching the charge-coupleddevice (CCD) detector. Through computer reconstruction, knowledge of the position of the wire can be used in conjunction with the intensity for each pixel located by the CCD detector to obtain the Laue diffraction pattern for that position of the wire. Depth resolution of the order of a tenth of a micrometer is possible, depending on the position of the x-ray-absorbing wire relative to the sample and detector.

This technique allows for a point-bypoint detailed determination of structure and 3D mapping. It can be used for extracting detailed information including microstructure and strain from a small, localized volume of a crystal. Particularly on the mesoscopic length scale, it allows for experimental determination of microstructural evolution that was previously only amenable to multiscale modeling and numerical simulations. The researchers provided examples of studies on grain structure in polycrystalline aluminum and orientation and strain measurements in cylindrically bent silicon.

GOPAL RAO

\section{Ordered Arrays of Aluminum \\ Nanoclusters Grown on Silicon}

Novel approaches for forming ordered structures of nanoclusters will likely play 